International Journal of Pure and Applied Mathematics

Volume 107 No. 4 2016, 971-981

ISSN: 1311-8080 (printed version); ISSN: 1314-3395 (on-line version)

url: http://www.ijpam.eu

doi: 10.12732/ijpam.v107i4.15

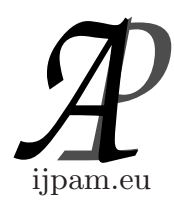

\title{
UNIQUENESS OF DIFFERENCE POLYNOMIALS OF MEROMORPHIC FUNCTIONS
}

\author{
Harina P. Waghamore ${ }^{1 \S}$, Rajeshwari S. ${ }^{2}$ \\ ${ }^{1,2}$ Department of Mathematics \\ Jnanabharathi Campus \\ Bangalore University \\ Bangalore, 560 056, INDIA
}

\begin{abstract}
In this paper, we study the zero distributions on the derivatives of q-shift difference polynomials of meromorphic functions with zero order and obtain two theorems that extend results of [3].
\end{abstract}

AMS Subject Classification: 30D35

Key Words: uniqueness, meromorphic function, difference polynomials, shared function

\section{Introduction}

In this paper, a meromorphic functions $f$ means meromorphic in the complex plane. If no poles occur, then $f$ reduces to an entire function. Throughout of this paper, we denote by $\rho(f)$ and $\rho_{2}(f)$ the order of $f$ and the hyper order of $f$ (Laine, 1993 and Yang and Yi, 2003). In addition, if $f-a$ and $g-a$ have the same zeros, then we say that $f$ and $g$ share the value $a \operatorname{IM}$ (ignoring multiplicities). If $f-a$ and $g-a$ have the same zeros, then we say that $f$ and $g$ share the value $a \mathrm{CM}$ (counting multiplicities). We assume that the reader is familiar with standard symbols and fundamental results of Nevanlinna Theory(Halburd Korhonen and Tohge; Laine, 1993 and Yang and Yi, 2003).

Given a meromorphic function $f(z)$, recall that $\alpha(z) \neq 0, \infty$ is a small function with respect to $f(z)$, if $T(r, \alpha)=S(r, f)$, where $S(r, f)$ is used to denote any quantity satisfying $S(r, f)=o(T(r, f))$, and $r \rightarrow \infty$ outside of a

Received: January 5, 2016

Published: May 7, 2016

${ }^{\S}$ Correspondence author (c) 2016 Academic Publications, Ltd. url: www.acadpubl.eu 
possible exceptional set of finite logarithmic measure.

Recently, K. Liu, X. Liu and T. B. Cao (2012) proved the following.

Theorem A. (Liu, Liu and Coa, 2012) Let $f$ be a transcendental entire function of $\rho_{2}(f)<1$. For $n \geq t(k+1)+1$, then $[P(f) f(z+c)]^{(k)}-\alpha(z)$ has infinitely many zeros.

Theorem B. (Liu, Liu and Coa, 2012) Let $f$ be a transcendental meromorphic function of $\rho_{2}(f)<1$, not a periodic function with period $c$. If $n \geq(t+1)(k+1)+1$, then $\left[f(z)^{n}\left(\Delta_{c} f\right)^{s}\right]^{(k)}-\alpha(z)$ has infinitely many zeros.

Theorem C. (Liu, Liu and Coa, 2012) Let $f$ be a transcendental meromorphic function of $\rho_{2}(f)<1$. For $n \geq t(k+1)+5$, then $[P(f) f(z+c)]^{(k)}-\alpha(z)$ has infinitely many zeros.

Theorem D. (Liu, Liu and Coa, 2012) Let $f$ be a transcendental meromorphic function of $\rho_{2}(f)<1$. If $n \geq(t+2)(k+1)+3+s$, then $\left[P(f)\left(\Delta_{c} f\right)^{s}\right]^{(k)}-$ $\alpha(z)$ has infinitely many zeros.

Theorem E. (Liu, Liu and Coa, 2012) Let $f$ and $g$ be a transcendental entire function of $\rho_{2}(f)<1, n \geq 2 k+m+6$. If $\left[f^{n}\left(f^{m}-1\right) f(z+c)\right]^{(k)}$ and $\left[g^{n}\left(g^{m}-1\right) g(z+c)\right]^{(k)}$ share the $1 C M$, then $f=t g$, where $t^{n+1}=t^{m}=1$.

Theorem F. (Liu, Liu and Coa, 2012) The conclusion of Theorem E is also valid, if $n \geq 5 k+4 m+12$. and $\left[f^{n}\left(f^{m}-1\right) f(z+c)\right]^{(k)}$ and $\left[g^{n}\left(g^{m}-1\right) g(z+c)\right]^{(k)}$ share the $1 \mathrm{IM}$.

In 2013, Harina P. Waghamore and Tanuja A. extend Theorem E and Theorem $\mathrm{F}$ to meromorphic functions.

Theorem G. (Harina P.W and Tanuja A, 2013) Let $f$ and $g$ be a transcendental meromorphic function with zero order. If $n \geq 4 k+m+8,\left[f^{n}\left(f^{m}-\right.\right.$ 1) $f(q z+c)]^{(k)}$ and $\left[g^{n}\left(g^{m}-1\right) g(q z+c)\right]^{(k)}$ share the $1 \bar{C} M$, then $f=t g$, where $t^{n+1}=t^{m}=1$.

Theorem H. (Harina P.W and Tanuja A, 2013) Let $f$ and $g$ be a transcendental meromorphic function with zero order. If $n \geq 5 k+4 m+17$, $\left[f^{n}\left(f^{m}-1\right) f(q z+c)\right]^{(k)}$ and $\left[g^{n}\left(g^{m}-1\right) g(q z+c)\right]^{(k)}$ share the 1 IM, then $f=t g$, where $t^{n+1}=t^{m}=1$. 
In this paper, we extend Theorem $\mathrm{G}$ and Theorem $\mathrm{H}$ to difference polynomials and obtain the following results.

Theorem 1. Let $f$ and $g$ be a transcendental meromorphic(resp. entire) function with zero order. If $n \geq 4 k+8(n \geq 2 k+6),[P(f) f(q z+c)]^{(k)}$ and $[P(g) g(q z+c)]^{(k)}$ share the 1 CM, then:

1. $f \equiv \operatorname{tg}$ for a constant $t$ such that $t^{d}=1$.

2. $f$ and $g$ satisfy the algebraic equation $R(f, g) \equiv 0$, where $R\left(w_{1}, w_{2}\right)=$ $P\left(w_{1}\right) w_{1}(q z+c)-P\left(w_{2}\right) w_{2}(q z+c)$.

Theorem 2. Let $f$ and $g$ be a transcendental meromorphic(resp. entire) function with zero order. If $n \geq 10 k+14(n \geq 5 k+12),[P(f) f(q z+c)]^{(k)}$ and $[P(g) g(q z+c)]^{(k)}$ share the 1 IM, then the conclusion of theorem 1 still holds.

\section{Some Lemmas}

In this section, we present some definitions and lemmas which will be needed in the sequel.

Lemma 2.1. (Halburd, Korhonen and Tohge, Theorem 5.1) Let $f(z)$ be a transcendental meromorphic function of $\rho_{1}(f)<1, \varsigma<1, \epsilon$ is enough small number. Then

$$
m\left(r, \frac{f(z+c)}{f(z)}\right)=o\left(\frac{T(r, f)}{r^{1-\varsigma-\epsilon}}\right)=S(r, f),
$$

for all $r$ outside of a set of finite logarithmic measure.Combining the proof of (Luo and Lin, 2011, Lemma 5) with Lemma 2.1, we can get the following Lemma 2.2.

Lemma 2.2. Let $f(z)$ be a transcendental entire functionof $\rho_{2}(f)<1$. If $F=P(f) f(z+c)$, then

$$
T(r, F)=T(P(f) f(z))+S(r, f)=(n+1) T(r, f)+S(r, f) .
$$

Lemma 2.3. (Liu, Liu and Cao, 2012, Lemma 2.5) Let $f(z)$ be a transcendental meromorphic function of $\rho_{2}(f)<1$. If $F=P(f) f(z+c)$, then

$$
(n-1) T(r, f)+S(r, f) \leq T(r, F) \leq(n+1) T(r, f)+S(r, f) .
$$


Lemma 2.4. (Zhang and Korhonen, 2010, Theorem 1.1) Let $f(z)$ be a transcendental meromorphic function of zero order. Then

$$
T(r, f(q z))=T(r, f(z))+S(r, f)
$$

on a set of logarithmic density 1.

The following lemma has little modifications of the original version (Theorem 2.1 of Chiang and Feng, 2008).

Lemma 2.5. Let $f(z)$ be a transcendental meromorphic function of finite order. Then

$$
T(r, f(z+c))=T(r, f)+S(r, f) .
$$

combining Lemma 2.4 with Lemma 2.5, we get the following result easily.

Lemma 2.6. Let $f(z)$ be a transcendental meromorphic function of zero order. Then

$$
T(r, f(q z+c))=T(r, f(z))+S(r, f)
$$

on a set of logarithmic density 1.

Lemma 2.7. (Yang and Hua, 1997, Lemma 3) Let $F$ and $G$ be non constant meromorphic functions. If $F$ and $G$ share $1 C M$, then one of the following three cases holds:

(i) $\max \{T(r, F), T(r, G)\} \leq N_{2}\left(r, \frac{1}{F}\right)+N_{2}(r, F)+N_{2}\left(r, \frac{1}{G}\right)+N_{2}(r, G)+$ $S(r, F)+S(r, G)$.

(ii) $F=G$.

(iii) $F . G=1$.

Lemma 2.8. (Xu an Yi, 2007, Lemma 2.3) Let $F$ and $G$ be non constant meromorphic function sharing the value 1 IM. Let

$$
H=\frac{F^{\prime \prime}}{F^{\prime}}-2 \frac{F^{\prime}}{F-1}-\frac{G^{\prime \prime}}{G^{\prime}}+2 \frac{G^{\prime}}{G-1} .
$$

If $H \neq 0$, then

$$
T(r, F)+T(r, G) \leq 2\left(N_{2}\left(r, \frac{1}{F}\right)+N_{2}(r, F)+N_{2}\left(r, \frac{1}{G}\right)+N_{2}(r, G)\right)
$$




$$
\begin{aligned}
& +3\left(\bar{N}(r, F)+\bar{N}\left(r, \frac{1}{F}\right)+\bar{N}(r, G)+\bar{N}\left(r, \frac{1}{G}\right)\right) \\
& +S(r, F)+S(r, G) .
\end{aligned}
$$

Lemma 2.9. Let $f(z)$ be a meromorphic function, and $p, k$ be positive integers. Then

$$
\begin{gathered}
T\left(r, f^{(k)}\right) \leq T(r, f)+k \bar{N}(r, f)+S(r, f) . \\
N_{p}\left(r, \frac{1}{f^{(k)}}\right) \leq T\left(r, f^{(k)}\right)-T(r, f)+N_{p+k}\left(r, \frac{1}{f}\right)+S(r, f) . \\
N_{p}\left(r, \frac{1}{f^{(k)}}\right) \leq k \bar{N}(r, f)+N_{p+k}\left(r, \frac{1}{f}\right)+S(r, f) .
\end{gathered}
$$

Lemma 2.10. Let $f$ and $g$ be a transcendental meromorphic function of zero order. If $n \geq k+6$ and

$$
[P(f) f(q z+c)]^{(k)}=[P(g) g(q z+c)]^{(k)}
$$

then $f=t g$, where $t^{n+1}=t^{m}=1$, and $f$ and $g$ satisfy the algebraic equation

$$
R\left(w_{1}, w_{2}\right)=P\left(w_{1}\right) w_{1}(q z+c)-P\left(w_{2}\right) w_{2}(q z+c) .
$$

Proof. From (2.10), we have

$$
P(f) f(q z+c)=P(g) g(q z+c)+Q(z)
$$

Where $Q(z)$ is a polynomial of degree atmost $k=1$. If $Q(z) \neq 0$, then we have

$$
\frac{P(f) f(z+c)}{Q(z)}=\frac{P(g) g(q z+c)}{Q(z)}+1
$$


From the second main theorem of Nevanlinna and by Lemma 2.2, we have

$$
\begin{aligned}
(n+1) T(r, f)= & T\left(r, \frac{P(f) f(q z+c)}{Q(z)}\right)+S(r, f) \\
\leq & \bar{N}\left(r, \frac{P(f) f(q z+c)}{Q(z)}\right)+\bar{N}\left(r, \frac{Q(z)}{P(f) f(q z+c)}\right) \\
+ & \bar{N}\left(r, \frac{Q(z)}{P(g) g(q z+c)}\right)+S(r, f) \\
\leq & \bar{N}(r, P(f))+\bar{N}(r, f(q z+c))+\bar{N}\left(r, \frac{1}{P(f)}\right) \\
& +\bar{N}\left(r, \frac{1}{f(q z+c)}\right) \\
& +\bar{N}\left(r, \frac{1}{g(z)}\right)+\bar{N}\left(r, \frac{1}{g(q z+c)}\right)+S(r, f)+S(r, g) \\
\leq & 4 T(r, f)+2 T(r, g)+S(r, f)+S(r, g) .
\end{aligned}
$$

Similarly as above, we have

$$
(n+1) T(r, g) \leq 4 T(r, g)+2 T(r, f)+S(r, f)+S(r, g) .
$$

Thus, we get

$$
(n+1)[T(r, f)+T(r, g)] \leq 6[T(r, f)+T(r, g)]+S(r, f)+S(r, g) .
$$

which is in contradiction with $n \geq k+6$. Hence, we get $Q(z) \equiv 0$, which implies that

$$
P(f) f(q z+c)=P(g) g(q z+c) .
$$

Set $h(z)=\frac{f(z)}{g(z)}$, we break the rest of the proof into two cases.

Case 1. Suppose $h(z)$ is a constant. Then by substituting $f=g h$ into (2.14), we obtain

$$
g(q z+c)\left[a_{n} g^{n}\left(h^{n+1}-1\right)+a_{n-1} g^{n-1}\left(h^{n}-1\right)+\ldots+a_{0}(h-1)\right] \equiv 0
$$

where $a_{n}(\neq 0), a_{n-1}, . ., a_{0}$ are complex constants. By the fact that $g$ is a transcendental entire functions, we have $g(q z+c) \not \equiv 0$. Hence, we obtain

$$
\left[a_{n} g^{n}\left(h^{n+1}-1\right)+a_{n-1} g^{n-1}\left(h^{n}-1\right)+\ldots+a_{0}(h-1)\right] \equiv 0 .
$$

Equation (2.16) implies that $h^{n+1}=1$ and $h^{i+1}=1$ when $a_{i} \neq 0$ for $i=$ $0,1, \ldots, n-1$. Therefore $h^{d}=1$, where $d=G C D\left(\lambda_{0}, \lambda_{1}, \ldots, \lambda_{n}\right)$. 
Case 2. Suppose that $h$ is not a constant, then we know by (2.14) that $f$ and $g$ satisfy the algebraic equation $R(f, g)=0$, where $R\left(w_{1}, w_{2}\right)=p\left(w_{1}\right) w_{1}(q z+$ $c)-p\left(w_{2}\right) w_{2}(q z+c)$.

Lemma 2.11. Let $f$ and $g$ be transcendental entire function of finite order. If $n \geq k+4$, and $[P(f) f(q z+c)]^{(k)}=[P(g) g(q z+c)]^{(k)}$ then the condition of Lemma 2.10 holds.

Proof. Substituting $\bar{N}(r, f)=\bar{N}(r, g)=0$ and proceeding as in the proof of Lemma 2.10, we get Lemma 2.11.

\section{Proof of the Theorem}

Proof of Theorem 1.1. Let $F=[P(f) f(q z+c)]^{(k)}$ and $G=[P(g) g(q z+c)]^{(k)}$. Thus $F$ and $G$ share the value 1 CM. From (2.7) and $f$ is a transcendental meromorphic function, then

$$
T(r, F) \leq T(r, P(f) f(q z+c))+k \bar{N}(r, f)+S(r, P(f) f(q z+c))
$$

combining (3.1) with Lemma 2.2, we have $S(r, F)=S(r, f)$. We also have $S(r, G)=S(r, g)$, from the same reason as above, from (2.8) we obtain

$$
\begin{aligned}
N_{2}\left(r \cdot \frac{1}{F}\right)= & N_{2}\left(r, \frac{1}{[P(f) f(q z+c)]^{(k)}}\right) \\
\leq & T(r, F)-T(r, P(f) f(q z+c)) \\
& +N_{k+2}\left(r, \frac{1}{P(f) f(q z+c)}\right)+S(r, f) .
\end{aligned}
$$

Thus, from Lemma 2.2 and (3.2) we get

$$
\begin{aligned}
(n+1) T(r, f)= & T(r, P(f) f(q z+c))+S(r, f) \\
\leq & T(r, F)-N_{2}\left(r, \frac{1}{F}\right) \\
& +N_{k+2}\left(r, \frac{1}{P(f) f(q z+c)}\right)+S(r, f)
\end{aligned}
$$

From (2.9), we obtain

$$
\begin{aligned}
N_{2}\left(r \cdot \frac{1}{F}\right) & \leq N_{k+2}\left(r, \frac{1}{P(f) f(q z+c)}\right)+S(r, f) \\
& \leq(k+2) N\left(r, \frac{1}{f}\right)+N\left(r, \frac{1}{f(q z+c)}\right)+k \bar{N}(r, f)+S(r, f) \\
& \leq(2 k+3) T(r, f)+S(r, f) .
\end{aligned}
$$


Similarly as above, we have

$$
\begin{gathered}
(n+1) T(r, g) \leq T(r, G)-N_{2}\left(r, \frac{1}{G}\right)+N_{k+2}\left(r, \frac{1}{P(g) g(q z+c)}\right)+S(r, g) \\
N_{2}\left(r \cdot \frac{1}{G}\right) \leq(2 k+3) T(r, g)+S(r, g) .
\end{gathered}
$$

If the (i) of Lemma 2.7 is satisfied implies that

$$
\begin{aligned}
\max \{T(r, F) T(r, G)\} \leq N_{2}\left(r, \frac{1}{F}\right)+N_{2}( & \left.r, \frac{1}{G}\right)+N_{2}(r, F) \\
& +N_{2}(r, G)+S(r, F)+S(r, G) .
\end{aligned}
$$

Thus, combining above with (3.3)-(3.6) we obtain

$$
\begin{aligned}
(n+1)\{T(r, f)+T(r, g)\} & \leq 2[N(r, f)+N(r, g)]+2 N_{k+2}\left(r, \frac{1}{P(f) f(q z+c)}\right) \\
& +2 N_{k+2}\left(r, \frac{1}{P(g) g(q z+c)}\right)+S(r, f)+S(r, g) \\
& \leq 2(2 k+4)\{T(r, f)+T(r, g)\}+S(r, f)+S(r, g) .
\end{aligned}
$$

Which is in contradiction with $n \geq 4 k+8$. Hence $F=G$ or $F G=1$. From Lemma 2.10, we get $f=t g$ for $t^{m}=t^{n+1}=1$ and $f$ and $g$ satisfy the algebraic equation $R(f, g)=0$, where $R\left(w_{1}, w_{2}\right)=P\left(w_{1}\right) w_{1}(q z+c)-p\left(w_{2}\right) w_{2}(q z+c)$.

Proof of Theorem 1.2. Let

$$
F=[P(f) f(q z+c)]^{(k)}, \quad G=[P(g) g(q z+c)]^{(k)} .
$$

Let $H$ be defined as in Lemma 2.8. Assume that $H \neq 0$, from (2.6) we get

$$
\begin{aligned}
T(r, F)+T(r, G) & \leq 2\left[N_{2}\left(r, \frac{1}{F}\right)+N_{2}\left(r, \frac{1}{G}\right)+N_{2}(r, F)+N_{2}(r, G)\right] \\
& +3\left[\bar{N}(r, F)+\bar{N}(r, G)+\bar{N}\left(r, \frac{1}{F}\right)+\bar{N}\left(r, \frac{1}{G}\right)\right] \\
& +S(r, F)+S(r, G) .
\end{aligned}
$$


Combining above with (3.3)-(3.6) and (2.9), we obtain

$$
\begin{aligned}
(n+1)[ & T(r, f)+T(r, g)] \leq T(r, F)+T(r, G)-N_{2}\left(r, \frac{1}{F}\right)-N_{2}\left(r, \frac{1}{G}\right) \\
& +N_{k+2}\left(r, \frac{1}{P(f) f(q z+c)}\right)+N_{k+2}\left(r, \frac{1}{P(g) g(q z+c)}\right) \\
& +S(r, f)+S(r, g) \\
& \leq 2\left(N_{2}(r, F)+N_{2}(r, G)\right)+2 N_{k+2}\left(r, \frac{1}{P(f) f(q z+c)}\right) \\
& +2 N_{k+2}\left(r, \frac{1}{P(g) g(q z+c)}\right)+3\left[\bar{N}\left(r, \frac{1}{F}\right)+\bar{N}\left(r, \frac{1}{G}\right)\right] \\
& +S(r, f)+S(r, g) \\
& \leq 2(2 k+4)\{T(r, f)+T(r, g)\}+3(2 k+2)\{T(r, f)+T(r, g)\} \\
& +S(r, f)+S(r, g) \\
& \leq(10 k+14)\{T(r, f)+T(r, g)\}
\end{aligned}
$$

which is a contradiction with $n \geq 10 k+14$. Thus we get $H \equiv 0$. The following proof is trivial, we give the complete proof. By integration for $H$ twice, we obtain

$$
F=\frac{(b+1) G+(a-b-1)}{b G+(a-b)}, \quad G=\frac{(a-b-1)-(a-b) F}{F b-(b+1)}
$$

which implies that $T(r, F)=T(r, G)+O(1)$. Since

$$
\begin{aligned}
T(r, F) & \leq T(r, P(f) f(q z+c))+S(r, f) \\
& \leq(n+1) T(r, f)+S(r, f),
\end{aligned}
$$

then $S(r, F)=S(r, f)$. So $S(r, G)=S(r, g)$ is. We distinguish into three cases as follows.

Case 1. $b \neq 0,-1$. If $a-b-1 \neq 0$, then by (3.8), we get

$$
\bar{N}\left(r, \frac{1}{F}\right)=\bar{N}\left(r, \frac{1}{F-\frac{a-b-1}{b+1}}\right)
$$

By the Nevanlinna second main theorem, (2.8) and (2.9), we have

$$
\begin{aligned}
(n+1) T(r, g) \leq & T(r, G)+N_{k+2}\left(r, \frac{1}{P(g) g(q z+c)}\right) \\
& -N\left(r, \frac{1}{G}\right)+S(r, g) \\
\leq & (k+1) T(r, g)+(2 k+2) T(r, f)+S(r, f)+S(r, g)
\end{aligned}
$$


Similarly, we get

$$
(n+1) T(r, f) \leq(k+1) T(r, f)+(2 k+2) T(r, g)+S(r, f)+S(r, g) .
$$

Thus from (3.10) and (3.11), then

$$
(n+1)\{T(r, f)+T(r, g)\} \leq(3 k+3)\{T(r, f)+T(r, g)\}+S(r, f)+S(r, g) \text {. }
$$

Which is in contradiction with $n \geq 10 k+14$. Thus $a-b-1=0$, then

$$
F=\frac{(b+1) G}{b G+1}
$$

using the same method as above, we get

$$
\begin{aligned}
(n+1) T(r, g) & \leq T(r, G)+N_{k}\left(r, \frac{1}{P(g) g(q z+c)}\right) \\
& -N\left(r, \frac{1}{G}\right)+S(r, g) \\
& \leq N_{k}\left(r, \frac{1}{P(g) g(q z+c)}\right)+\bar{N}\left(r, \frac{1}{G+\frac{1}{b}}\right)+S(r, g) \\
& \leq(k+1) T(r, g)+S(r, g) .
\end{aligned}
$$

Which is a contradiction.

Case 2. $b=0, a \neq 1$. From (3.8), we have

$$
F=\frac{G+a-1}{a}
$$

Similarly, we also can get a contradiction. Thus $a=1$ follows, it implies that $F=G$.

Case 3. $b=-1, a \neq-1$. From (3.8), we obtain

$$
F=\frac{a}{a+1-G}
$$

Similarly, we can get a contradiction, $a=-1$ follows. Thus, we get $F \cdot G=1$.

From Lemma 2.10, we get $f=t g$ for $t^{m}=t^{n+1}=1$, and $f$ and $g$ satisfy the algebraic expression $R(f, g)=0$, where

$$
R\left(w_{1}, w_{2}\right)=P\left(w_{1}\right) w_{1}(q z+c)-P\left(w_{2}\right) w_{2}(q z+c)
$$

Thus, we have completed the proofs. 


\section{References}

[1] Y.-M. Chiang and S.-J. Feng, On the Nevanlinna characteristic of $f(z+\eta)$ and difference equations in the complex plane, Ramanujan J. 16 (2008), no. 1, 105-129.

[2] R. Halburd, R. Korhonen and K. Tohge, Holomorphic curves with shift-invariant hyperplane preimages, Trans. Amer. Math. Soc. 366 (2014), no. 8, 4267-4298.

[3] H. P. Waghamore and A. Tanuja, Uniqueness of difference polynomials of meromorphic functions, Bull. Calcutta Math. Soc. 105 (2013), no. 3, 227-236.

[4] W. K. Hayman, Meromorphic functions, Oxford Mathematical Monographs, Clarendon Press, Oxford, 1964.

[5] I. Laine, Nevanlinna theory and complex differential equations, de Gruyter Studies in Mathematics, 15, de Gruyter, Berlin, 1993.

[6] Liu Kai; Liu Xinling and Cao Tingbin (2012): Some results on zeros distributions and uniqueness of derivatives of difference polynomials, Ann. Polon. Math., 109, 137.

[7] X. Luo and W.-C. Lin, Value sharing results for shifts of meromorphic functions, J. Math. Anal. Appl. 377 (2011), no. 2, 441-449.

[8] A. Z. Mohon'ko, The Nevanlinna characteristics of certain meromorphic functions, Teor. Funkciŭ Funkcional. Anal. i Priložen. No. 14 (1971), 83-87.

[9] J. Xu and H. Yi, Uniqueness of entire functions and differential polynomials, Bull. Korean Math. Soc. 44 (2007), no. 4, 623-629.

[10] C.-C. Yang and H.-X. Yi, Uniqueness theory of meromorphic functions, Mathematics and its Applications, 557, Kluwer Acad. Publ., Dordrecht, 2003.

[11] C.-C. Yang and X. Hua, Uniqueness and value-sharing of meromorphic functions, Ann. Acad. Sci. Fenn. Math. 22 (1997), no. 2, 395-406.

[12] J. Zhang and R. Korhonen, On the Nevanlinna characteristic of $f(q z)$ and its applications, J. Math. Anal. Appl. 369 (2010), no. 2, 537-544. 
